\title{
Hypovitaminosis D, oral potentially malignant disorders, and oral squamous cell carcinoma: a systematic review
}

\author{
Andrea Maturana-Ramírez ${ }^{1,2}$, Juan Aitken-Saavedra ${ }^{1,3}$, Arantxa Ladrón de Guevara-Benítez ${ }^{4}$, Iris Espinoza- \\ Santander ${ }^{1}$ \\ ${ }^{1}$ Department of Oral Pathology and Medicine, Faculty of Dentistry, Universidad de Chile, Santiago, Chile \\ ${ }^{2}$ Therapeutic Diagnostic Center Odontology and Pathological Anatomy Service, Hospital Complex San Jose, Santiago, Chile \\ ${ }^{3}$ San Camilo Hospital, San Felipe, Chile \\ ${ }^{4}$ Undergraduate, Faculty of Dentistry, Universidad de of Chile, Santiago, Chile
}

Correspondence:

Department of Oral Pathology and Medicine

Faculty of Dentistry, Universidad de Chile

Postal address: 8380492 Olivos 943, Independencia, Santiago, Chile

amaturana@odontologia.uchile.cl

Received: 06/08/202

Accepted: 07/02/2022

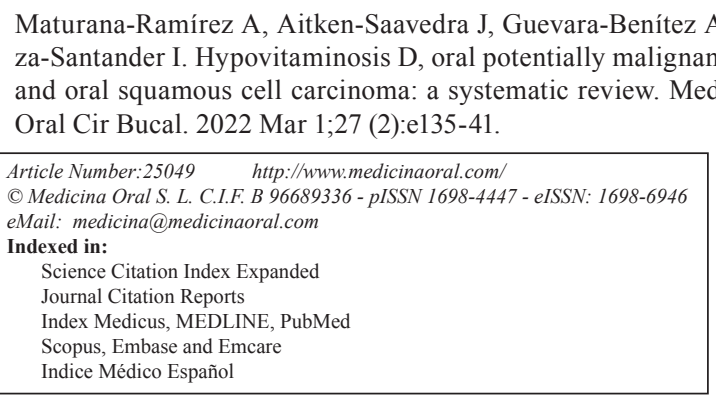

\begin{abstract}
Background: Oral squamous cell carcinoma (OSCC) and potentially malignant oral disorders (OPMDs) could be associated with low levels of vitamin $\mathrm{D}$. This systematic review aimed to determine the relationship between serum levels of vitamin D with OPMDs and OSCC.

Material and Methods: This review was conducted according to Cochrane guidelines (PROSPERO CRD42020207382) on literature retrieved from the PubMed, Cochrane, and Web of Science databases. The antecedents extracted were study design, methodology, sample (country, number of patients, age, and sex), oral manifestations (type of lesion, location, prevalence, and follow-up), serum vitamin D levels or use of vitamin D supplements, results, and conclusions. Results: Twelve articles were selected. Some of the most relevant findings were alterations in vitamin D could favor the progress of OPMDs to OSCC. Higher levels of vitamin D can increase levels of anti-inflammatory mediators, $\mathrm{CD} 4+\mathrm{T}$ lymphocytes and CD8+ T lymphocytes and CD3 $+\mathrm{T}$ lymphocytes in intratumoral tissue. The normalization of vitamin D levels in patients with OSCC can increased cytotoxic activity of natural killer cells, favoring antitumor immune response. Vitamin D supplemented can lower adverse effects associated with chemotherapy like mucositis and pain. Tobacco can increase risk of developing OSCC altering vitamin D levels.

Conclusions: Hypovitaminosis D could increase risk of developing OSCC from OPMDs, thus altering the immune response and it is associated with a lower survival rate in patients with OSCC, a greater recurrence of tumors in patients who underwent surgical treatment, and an increase in adverse reactions to chemotherapy. The use of vitamin D supplements can be a complement to primary therapy to prevent the recurrence of lesions and reduce adverse events associated with treatment.
\end{abstract}

Key words: Oral squamous cell carcinoma (OSCC), Oral potentially malignant disorders (OPMDs), Vitamin D, Oral cancer. 


\section{Introduction}

Cancer is currently the second leading cause of death in the world and is expected to take first place in many countries by 2030 (1). Oral squamous cell carcinoma (OSCC) corresponds to $90 \%$ of oral cancers. It features an aggressive pathology, usually with a poor prognosis due to its late diagnosis since the lesions are usually detected at stage III or IV (2), radicalizing the treatment options and negatively impacting the quality of life of patients. Five-year survival figures for oral cancer have not improved (50-60\% overall survival) (3). Oral potentially malignant disorders (OPMDs) are characterized by morphological alterations indicating an increased potential for malignant transformation. They are further associated with a greater risk of developing cancer, i.e., OSCC, at any site of the oral mucosa (4). Thus, although some lesions present a greater risk of progressing to OSCC, the risk is also in apparently healthy mucosa, in fact, may arise at a site distant to an existing premalignant lesion $(5,6)$.

Vitamin D as having different anticancer effects (7). Vitamin D is a steroid hormone that has two main forms in humans, vitamin D3 or cholecalciferol, which is synthesized in the skin after exposure to sunlight or ultraviolet light, and vitamin D2 or ergocalciferol, which is obtained through the irradiation of plants, plant materials, or food (8). Serum vitamin D is metabolized in the liver and then in the kidneys to obtain its active metabolite, $1,25(\mathrm{OH}) 2 \mathrm{D}$, which binds to vitamin $\mathrm{D}$ receptors (VDR) widely distributed in different tissues, promoting cell differentiation, inhibiting the proliferation of carcinogenic cells, and promoting anti-inflammatory, pro-apoptotic, and antiangiogenic effects (9). On the other hand, there have suggested a relationship between hypovitaminosis D with an increased risk of cancer incidence and associated mortality (9). Optimal vitamin D levels have been associated with a lower likelihood of developing OSCC and longer survival after diagnosis (10). Although a possible association between hypovitaminosis D and the potential for malignancy has been suggested, the evidence is scarce and contradictory. The objective of this systematic review was to establish the association between hypovitaminosis $\mathrm{D}$ with potentially malignant disorders and OSCC.

\section{Material and Methods}

This systematic review was conducted according to the guidelines of the Cochrane Handbook for Systematic Reviews of Interventions, following the four-phase flow chart of the Preferred Reporting Items for Systematic Reviews and Meta-Analyses Statement and it is registered in the PROSPERO International Prospective Registry of Systematic Reviews under Code Number CRD42020207382. Reviewers conducted the review according to PubMed (National Library of Medicine),
Cochrane (Elsevier), and Web of Science (Thomson Reuters) databases. In addition, the reference lists of selected articles were further searched for additional articles. Articles published in English, Spanish, and Portuguese between 2009 and 2020 were considered. The search strategy is described in (Supplement 1).

All studies reported in English, Portuguese, or Spanish that met the inclusion or problem, intervention, comparison, and outcome criteria were analyzed. Clinical trials and clinical, population, case-control, and cohort studies were included. The studies should have been carried out in humans over 18 years of age where measurements of serum vitamin D levels were obtained, or vitamin D supplementation was included in the treatment of patients with a histopathological diagnosis of a potentially malignant disorder or OSCC. Animal studies, in vitro or in vivo studies, and previous systematic reviews were excluded. When the search results were obtained, the titles and abstracts were assessed to select the articles that showed the greatest agreement, which were subsequently retrieved in full to verify the degree of compliance with the eligibility criteria (inclusion and exclusion criteria). The antecedents extracted from each study were language, study design, methodology and eligibility criteria, sample (country, number of patients, age, and sex), oral manifestations (type of lesion, location, prevalence, and follow-up), vitamin D serum evaluation or use of vitamin D supplements, results, and conclusions. A random-effects model was used for analysis. Due to the high degree of heterogeneity in terms of study design and methodology, performing a quantitative analysis of the meta-analysis was considered inappropriate.

\section{Results}

A total of 886 articles were retrieved through the indicated platforms, and 4 articles were identified through manual methods. Of the 886 articles, 107 duplicates were removed. The remaining 783 articles were filtered by title, abstract, and inclusion and eligibility criteria. After an exhaustive evaluation, 12 texts were finally selected for analysis (11-21). The flow chart for the methodology is shown in Fig. 1.

The analysis of the 12 articles is summarized in Table 1. Four studies were conducted in Asia $(11,12,14,17), 4$ in Europe $(10,13,15,18), 4$ in North America (United States) (16,19-21), Six were case-control studies (11-13,15-17), 3 were cohort $(10,14,18)$, and 3 were clinical trials (19-21). The methods of measuring serum vitamin $\mathrm{D}$ levels included electrochemiluminescence $(n=1)(12)$, chemiluminescence $(\mathrm{n}=2)(13,14)$, enzyme-linked immunosorbent assays $(\mathrm{n}=1)(17)$, liquid chromatography $(\mathrm{n}=2)$ $(10,18)$, and radioimmunoassay $(\mathrm{n}=1)(17)$. Of the articles that specified the age of the patients $(10,16-18,20,21)$, the age range was between 20 and 92 years, whereas that for patients with OPMDs was between 37.2 and 60.7 years. 


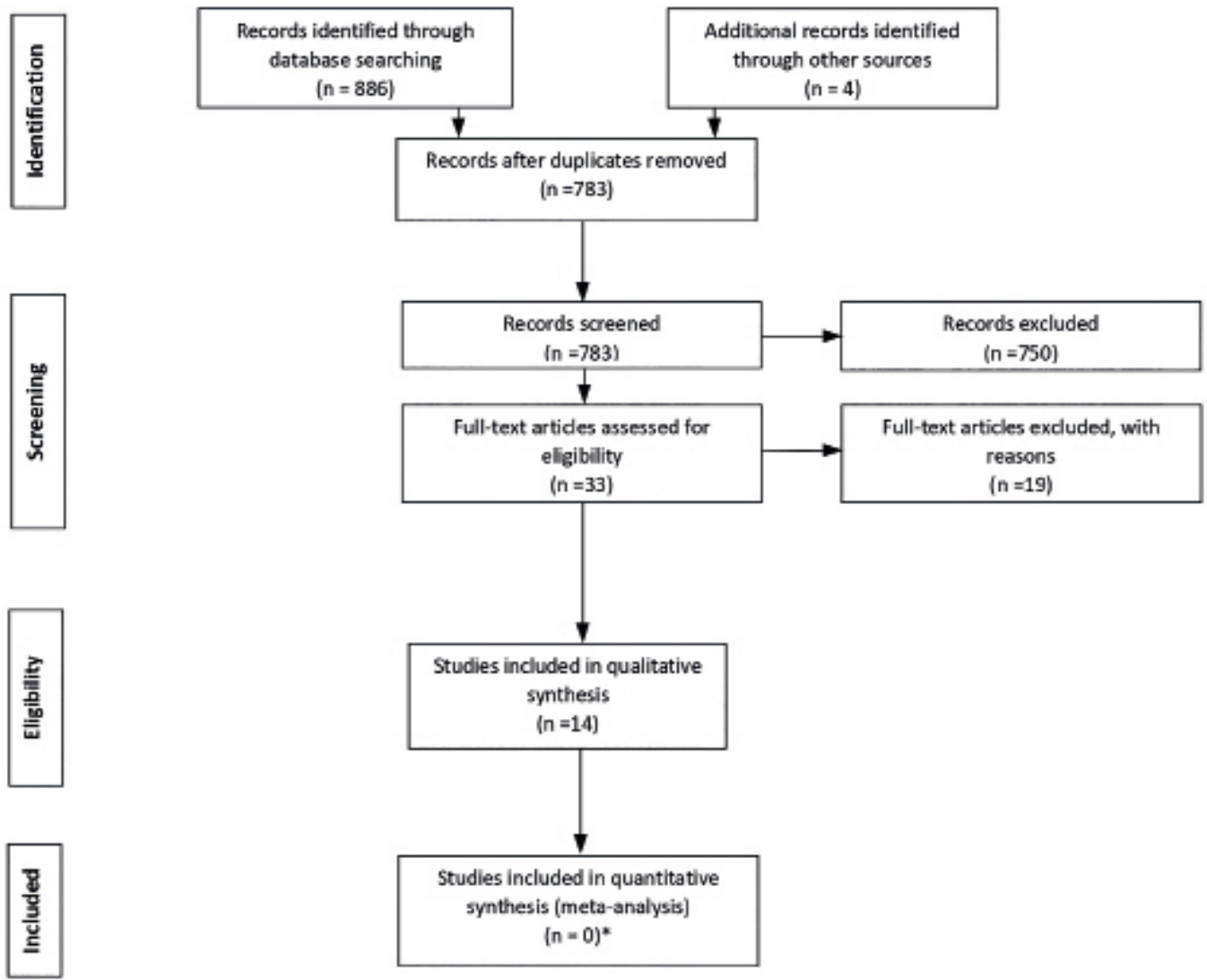

Fig. 1: Search flowchart according to the PRISMA Statement.

Table 1: Review's included articles.

\begin{tabular}{|c|c|c|c|c|c|}
\hline Authors & Country & Study type & Cases (n) & Controls (n) & Diagnosis \\
\hline Udeabor et al. (11) & Saudi Arabia & Case control & 51 & 113 & OSCC \\
\hline Bahramian et al. (12) & Iran & Case control & 18 & 18 & OLP \\
\hline Bochen et al. (13) & Germany & Case control & 231 & 232 & $\mathrm{HNC}$ - oral cancer \\
\hline Anand et al. (14) & India & Cohort & 197 & 95 & PMD and OSCC \\
\hline Fanidi et al. (10) & Multicentric Europe & Cohort & 110 & 443 & $\mathrm{HNC}$ - oral cancer \\
\hline Grimm et al. (15) & Germany & Case control & 42 & 5 & PMD and OSCC \\
\hline Young et al. (16) & United States & Case control & 93 & 30 & PMD and OSCC \\
\hline Zhang et al. (17) & China & Case control & 70 & 70 & OSCC \\
\hline Orell-Kotikangas et al. (18) & Finland & Cohort & 65 & N.E. & HNC - oral cancer \\
\hline Walker et al. (19) & United States & Clinical trial & 53 & 23 & $\mathrm{HNC}$ \\
\hline Walsh et al. (20) & United States & Clinical trial & 16 & 16 & HNC - oral cancer \\
\hline Lipworth et al. (21) & United States & Clinical trial & 405 & 2080 & HNC - oral cancer \\
\hline
\end{tabular}

HNC: head and neck cancer, OSCC: oral squamous cell carcinoma, PMOD: potentially malignant oral disorders, OLP: oral lichen planus, N.S.: not specify. 
In patients with OSCC, the age range was between 48 and 63 years. In the articles that specified the distribution by sex $(10-16,18,20,21)$, male patients were predominant, ranging between $57.1 \%$ and $82 \%$ of the sample population. In those with a diagnosis of oral lichen planus (OLP) (12), the female sex was predominant, between $61.1 \%$ and $70 \%$ of the population. Of the articles that specified the lesion site $(n=6)(11,13-$ $15,18,20)$, for OSCC, the most frequent sites were the floor of the mouth, jugal mucosa, ridge, and tongue. Regarding the habits of the patients, 4 articles listed smoking $(10,17,18,21)$, and of these, 3 also listed alcohol consumption $(10,17,21)$. Three articles associated these habits with serum vitamin D levels $(10,18,21)$, and 1 identified difference in smokers with low vitamin D levels (18). Two articles observed a higher risk of developing cancer in smokers with low vitamin D $(10,21)$. Of the articles that considered patients with OSCC (n =11) $(10,11,13,14,21), 5$ exclusively considered patients with OSCC (11,14,15-17), whereas 6 also included other locals of squamous cell carcinoma (SCC) such as oropharynx, nasopharynx, hypopharynx, and larynx $(10,13,18-21)$. The results of the 5 articles that specified the measurement methods and values used for vitamin $\mathrm{D}$ are summarized in Table 2.

Three articles evaluated variations in the immune response of the subjects according to their vitamin D levels $(13,16,19)$. Young et al. indicated that patients supplemented weekly with $12 \mu \mathrm{g}$ of 1,25(OH)2D 3 in a 3 -week period had higher levels of anti-inflammatory mediators such as adiponectin and a decrease in pro-inflammatory mediators such as IL-6, IL-17, and leptin (16). Bochen et al. and Walsh et al. indicated an increase in CD4+ T lymphocytes and CD8+ T lymphocytes in the intratumoral tissue of patients with SCC who received vitamin D supplementation $(13,20)$. Bochen et al. evaluated other cell populations, observing an increase in $\mathrm{CD} 3+\mathrm{T}$ lymphocytes, natural killer cells, macrophages, and M1 macrophages in intratumoral tissue (13).
Grimm et al. evaluated VDR in relation to its expression in oral keratinocytes in case and control patients, showing that patients with OLP had an up to $50 \%$ lower expression rate of receptors in the oral mucosa compared with those without lesions (15). Furthermore, Anand et $a l$. associated low vitamin D levels with greater severity of post-chemotherapy adverse reactions such as edema, erythema, ulcers, and pain (14). They found that increasing serum vitamin D levels mitigated the adverse effects associated with chemotherapy in patients with OSCC such as oral mucositis and pain and improved swallowing and quality of life.

\section{Discussion}

Our results showed that Asia is one of the continents with the highest number of publications, probably because it has a high incidence of OSCC in the world (22). Studies on patients with OPMDs used different methodologies to histopathologically characterize oral epithelial dysplasias. In 2017, WHO presented two ways of classifying dysplasias: graduation of dysplasia into "mild," "moderate," and "severe" categories (WHO dysplasia grade) and a binary system of "low" and "high" grade (4). The use of the latter classification could simplify the comparison between patients and avoid biases. Indeed, the use of different criteria to assess the degree of dysplasia and vitamin D levels makes the comparison of the different articles difficult.

Patients with OPMDs were over 40 years of age (23), and the frequency of OSCC increased between the fifth and sixth decades of life $(24,25)$. OSCC and OPMDs are more frequent in men, except in OLP, which affects more women (26). Localization in both OPMDs and OSCC mostly involved the tongue, floor of the mouth, and alveolar mucosa (27). In accordance with habits, tobacco could alter serum vitamin D levels through smoking by-products such as lead and cadmium (28). Moreover, tobacco use can promote alterations in the expressions of CYP24A1 and CYP27B1, which affect

Table 2: Evaluation of vitamin D levels.

\begin{tabular}{|c|c|c|c|c|c|c|}
\hline Autores & $\begin{array}{l}\text { Technique } \\
\text { used }\end{array}$ & $\begin{array}{c}\text { Metabolite Evalua- } \\
\text { ted }\end{array}$ & $\begin{array}{l}\text { Vitamin D Serum } \\
\text { levels of considered } \\
\text { normal }(\mathrm{ng} / \mathrm{ml})\end{array}$ & $\begin{array}{l}\text { Controls } \\
(\mathrm{ng} / \mathrm{ml})\end{array}$ & $\begin{array}{c}\text { Cases } \\
(\mathrm{ng} / \mathrm{ml})\end{array}$ & $p$ \\
\hline Bahramian et al. (12) & ECLIA & Calcidiol $(25(\mathrm{OH}) \mathrm{D})$ & N.E. & $30,7 \pm 20,38$ & $36,45 \pm 15,33$ & 0,346 \\
\hline Bochen et al. (13) & CLIA & Calcidiol $(25(\mathrm{OH}) \mathrm{D})$ & 30 & 21,8 & 11,1 & 0,0001 \\
\hline Anand et al. (14) & CLIA & Calcidiol $(25(\mathrm{OH}) \mathrm{D})$ & 30 & $\begin{array}{c}68,4 \% \\
(12-29 \mathrm{ng} / \mathrm{ml})\end{array}$ & $\begin{array}{c}76,3 \% \\
(6-30 \mathrm{ng} / \mathrm{ml})\end{array}$ & 0,002 \\
\hline Grimm et al. (15) & RIA & Calcidiol $(25(\mathrm{OH}) \mathrm{D})$ & 36 & N.S. & 12,2 & N.S. \\
\hline Zhang et al. (17) & ELISA & Calcidiol $(25(\mathrm{OH}) \mathrm{D}$ & N.S. & 24 & 24 & $p>0,05$ \\
\hline
\end{tabular}

ECLIA: Electrochemiluminescence, CLIA: Chemiluminescence, ELISA: Enzyme-Linked Immunosorbent Assay, RIA: Radioimmunoassay, CCC: Head and Neck Cancer, OC: Oral Cancer, N.S: Not Specified. 
the enzymes that regulate circulating vitamin D levels. Furthermore, aging of the skin, a product of the free radicals in tobacco, would impact the main source of vitamin D in the body (29).

Optimal serum vitamin D levels vary according to life stage, race, ethnicity, sex, and others. No consensus was observed in the categorization of normal serum vitamin $\mathrm{D}$ values, but some authors agreed that vitamin D levels above $30 \mathrm{ng} / \mathrm{ml}$ are sufficient for normal function $(13,14)$. Levels over $30 \mathrm{ng} / \mathrm{ml}$ could be necessary to maximize the effects of vitamin D on calcium, bone, and muscle metabolism. However, a range between 150 and $200 \mathrm{ng} /$ $\mathrm{ml}$ would be associated with intoxication and adverse effects (30-32). Between 1,000 and 10,000 IU may be needed to normalize serum levels in adult patients to 30 $\mathrm{ng} / \mathrm{ml}$. However, patients with conditions such as obesity, cancer, disability, or gastrointestinal problems may require around 4,000 IU/day $(29,30,33)$. Recommended Dietary Allowances (RDAs) for vitamin D, are $600 \mathrm{IU} / \mathrm{d}$ for 1-70 years' persons and $800 \mathrm{IU} / \mathrm{d}$ for ages 71 year and older, corresponding to a serum 25-hydroxyvitamin D level of at least $20 \mathrm{ng} / \mathrm{ml}$ (50 nmol/liter) (34). According to Haines et al. (35), in patients with vitamin D deficiency, a cumulative dose of at least 600,000 IU administered over several weeks may be necessary to replenish vitamin D stores and large single doses of 300,000 to 500,000 IU should be avoided. Furthermore, the decision of otherwise healthy young adults to take vitamin D at doses of $2000 \mathrm{IU} /$ day or less is unlikely to cause harm. For patients who are not at risk of developing vitamin D deficiency, sensitive sun exposure is an inexpensive way to maintain vitamin D stores.

Hypovitaminosis D increased the susceptibility of patients with OPMDs to progression to OSCC (14) and was related to an increase of adverse effects associated with OSCC treatment, increased recurrence of neoplastic lesions, and worse prognosis for patients (20). The normalization of vitamin D levels in patients with SCC increased the cytotoxic activity of natural killer cells, favoring antitumor immune response (13). In OSCC patients with given vitamin D supplementation, proinflammatory profiles similar to patients with OPMDs given supplementation were observed, demonstrating a relationship between tissue and plasma changes. A reduction in anti-inflammatory mediators such as adiponectin and an increase in pro-inflammatory mediators such as IL-6, IL-17, and leptin were related to low serum vitamin D levels (16). In patients with $\mathrm{CCC}$, defects in the maturation of immune cells and increased levels of immature forms of myeloid suppressor cells or CD34+ progenitor cells were also associated with lower vitamin D levels. These phenomena could explain the susceptibility of tissues to develop malignant neoplasms from OPMDs (16).

Supplementation with D3 or D2 resulted in increased serum $25(\mathrm{OH}) \mathrm{D}$ levels, with a peak in the values at day 14. Increasing vitamin D levels alleviated the adverse effects associated with chemotherapy such as oral mucositis, pain, and difficulty in swallowing. This could be because vitamin D possibly stimulates the differentiation and epithelialization in the cells of the oral mucosa (14). A VDR Tt genotype presents a significant risk for the development of OSCC, whereas a VDR tt genotype would be a protective factor. In addition, the $\mathrm{T}$ allele was found in all patients diagnosed with OSCC or had tumor diameters larger than $4 \mathrm{~cm}$ (36). The VDR Taq I genotype with the $\mathrm{t}$ allele has been associated with a reduced risk of developing lung cancer due to tobacco use of up to $28 \%$ (37).

The current available literature that relates hypovitaminosis D with OPMDs and OSCC is scarce, and few authors have described measurement methods used to determine vitamin D levels. Moreover, since no standardization has been implemented for vitamin D measurement both in supplemented patients and in those who do not, the risk of bias due to physiological variations is high. Despite the limitations of our study, to our knowledge, this is the first literature review establishing an association between hypovitaminosis D and OPMDs and OSCC. It is necessary to mention that due to the different types of study designs, the diversity of the methodological quality of each one and the high percentage $(85 \%)$ of observational approaches, the level of evidence is still low. In addition, the publishing bias, can overestimate the real effect of vitamin D. Besides, low number of studies included $(n=12)$ limited the extrapolation of the results. Moreover, whether genetically higher cancer risk can influence vitamin D level, namely the reverse causation, remains unknown. Further studies are needed to physiologically clarify the mechanism by which vitamin D supplements modify the immune response of patients, in addition to studies that explain the mechanism by which hypovitaminosis $\mathrm{D}$ increases the susceptibility to developing OSCC and how supplementation could favor the normalization of the immune system.

The findings of this review suggest that hypovitaminosis D favors the progression from OPMDs to OSCC and is associated with lower survival in patients with SCC and OSCC, greater recurrence of tumors in patients receiving surgical treatment, and increased adverse reactions associated with chemotherapy. This review has identified a trend correlating hypovitaminosis D and the expression of certain VDR genotypes, which can indicate the risk for malignant progression in patients with OPMDs due to alterations in immune response. Tobacco use, in addition to being a risk factor associated with the development of different types of cancer, was identified as a negative factor on vitamin D levels favoring the progress from OPMDs to OSCC. The use 
of vitamin D supplements in patients with OSCC could be used as an adjunct to primary therapy to prevent recurrence of lesions and reduce adverse reactions associated with treatment. Regarding other cancers, new evidence also sheds light on repurposing vitamin $\mathrm{D}$ as a potential therapeutic agent for gastric cancer prevention and treatment for example (38).

\section{References}

1. Bray F, Ferlay J, Soerjomataram I, Siegel RL, Torre LA, Jemal A. Global cancer statistics 2018: GLOBOCAN estimates of incidence and mortality worldwide for 36 cancers in 185 countries. CA Cancer J Clin. 2018;68:394-424.

2. Seoane-Romero JM, Vázquez-Mahía I, Seoane J, Varela-Centelles P, Tomás I, López-Cedrún JL. Factors related to late-stage diagnosis of oral squamous cell carcinoma. Med Oral Patol Oral Cir Bucal. 2012;17:e35-40.

3. Gupta S, Kong W, Peng Y, Miao Q, Mackillop WJ. Temporal trends in the incidence and survival of cancers of the upper aerodigestive tract in Ontario and the United States. Int J Cancer. 2009;125:2159-65. 4. El-Naggar AK, Chan JKC, Takata T, Grandis JR, Slootweg PJ. The fourth edition of the head and neck World Health Organization blue book: editors' perspectives. Hum Pathol. 2017;66:10-2.

5. Speight PM, Khurram SA, Kujan O. Oral potentially malignant disorders: risk of progression to malignancy. Oral Surg Oral Med Oral Pathol Oral Radiol. 2018;125:612-27.

6. Warnakulasuriya S. Oral potentially malignant disorders: A comprehensive review on clinical aspects and management. Oral Oncol. 2020;102:104550.

7. Feldman D, Krishnan AV, Swami S, Giovannucci E, Feldman BJ. The role of vitamin $\mathrm{D}$ in reducing cancer risk and progression. Nat Rev Cancer. 2014;14:342-57.

8. Lips P. Vitamin D physiology. Prog Biophys Mol Biol. 2006;92:4-8. 9. Krishnan AV, Feldman D. Mechanisms of the anti-cancer and antiinflammatory actions of vitamin D. Annu Rev Pharmacol Toxicol. 2011;51:311-36

10. Fanidi A, Muller DC, Midttun Ø, Ueland PM, Vollset SE, Relton $\mathrm{C}$, et al. Circulating vitamin $\mathrm{D}$ in relation to cancer incidence and survival of the head and neck and oesophagus in the EPIC cohort. Sci Rep. 2016;6:36017.

11. Udeabor SE, Albejadi AM, Al-Shehri WAK, Onwuka CI, AlFathani SY, Al Nazeh AA, et al. Serum levels of 25-hydroxy-vitamin $\mathrm{D}$ in patients with oral squamous cell carcinoma: Making a case for chemoprevention. Clin Exp Dent Res. 2020;6:428-32.

12. Bahramian A, Bahramian M, Mehdipour M, Falsafi P, Khodadadi S, Dabaghi Tabriz F, et al. Comparing Vitamin D Serum Levels in Patients with Oral Lichen Planus and Healthy Subjects. J Dent (Shiraz). 2018;19:212-6.

13. Bochen F, Balensiefer B, Körner S, Bittenbring JT, Neumann F, Koch A, et al. Vitamin D deficiency in head and neck cancer patients - prevalence, prognostic value and impact on immune function. Oncoimmunology. 2018;7:e1476817.

14. Anand A, Singh S, Sonkar AA, Husain N, Singh KR, Singh S, et al. Expression of vitamin D receptor and vitamin D status in patients with oral neoplasms and effect of vitamin D supplementation on quality of life in advanced cancer treatment. Contemp Oncol (Pozn). 2017; $21: 145-51$

15. Grimm M, Cetindis M, Biegner T, Lehman M, Munz A, Teriete $\mathrm{P}$, et al. Serum vitamin D levels of patients with oral squamous cell carcinoma (OSCC) and expression of vitamin D receptor in oral precancerous lesions and OSCC. Med Oral Patol Oral Cir Bucal. 2015;20:e188-95.

16. Young MR, Levingston C, Johnson SD. Cytokine and Adipokine Levels in Patients with Premalignant Oral Lesions or in Patients with Oral Cancer Who Did or Did Not Receive 1 1 ,25Dihydroxyvitamin D3 Treatment upon Cancer Diagnosis. Cancers (Basel). 2015;25:1109-24.
17. Zhang H, Lu H, Shrestha C, Feng Y, Li Y, Peng J, et al. In serum, higher parathyroid hormone but not lower vitamin $\mathrm{D}$ is associated with oral squamous cell carcinoma. Curr Oncol. 2015;22:e259-63. 18. Orell-Kotikangas H, Schwab U, Österlund P, Saarilahti K, Mäkitie O, Mäkitie AA. High prevalence of vitamin D insufficiency in patients with head and neck cancer at diagnosis. Head Neck. 2012;34:1450-5.

19. Walker DD, Reeves TD, de Costa AM, Schuyler C, Young MR. Immunological modulation by $1 \alpha, 25$-dihydroxyvitamin D3 in patients with squamous cell carcinoma of the head and neck. Cytokine. 2012;58:448-54

20. Walsh JE, Clark AM, Day TA, Gillespie MB, Young MR. Use of alpha,25-dihydroxyvitamin D3 treatment to stimulate immune infiltration into head and neck squamous cell carcinoma. Hum Immunol. 20101;71:659-65.

21. Lipworth L, Rossi M, McLaughlin JK, Negri E, Talamini R, Levi $\mathrm{F}$, et al. Dietary vitamin D and cancers of the oral cavity and esophagus. Ann Oncol. 2009;20:1576-81.

22. Ferlay J, Colombet M, Soerjomataram I, Mathers C, Parkin $\mathrm{DM}$, Piñeros $\mathrm{M}$, et al. Estimating the global cancer incidence and mortality in 2018: GLOBOCAN sources and methods. Int J Cancer. 2019;15:1941-53.

23. Balsaraf S, Bhambal A, Chole R. Study of oral potentially malignant disorders related to various risk factors amongst the patients' attending hospitals in Bhopal, India. Med Pharm Rep. 2019;92:66-71. 24. Ellington TD, Henley SJ, Senkomago V, O'Neil ME, Wilson RJ, Singh S, et al. Trends in Incidence of Cancers of the Oral Cavity and Pharynx - United States 2007-2016. MMWR Morb Mortal Wkly Rep. 2020;69:433-8.

25. Montero PH, Patel SG. Cancer of the oral cavity. Surg Oncol Clin N Am. 2015;24:491-508.

26. Varghese SS, George GB, Sarojini SB, Vinod S, Mathew P, Mathew DG, et al. Epidemiology of Oral Lichen Planus in a Cohort of South Indian Population: A Retrospective Study. J Cancer Prev. 2016;21:55-9.

27. Pires FR, Ramos AB, Oliveira JB, Tavares AS, Luz PS, Santos TC. Oral squamous cell carcinoma: clinicopathological features from 346 cases from a single oral pathology service during an 8-year period. J Appl Oral Sci. 2013;21:460-7.

28. Mousavi SE, Amini H, Heydarpour P, Amini Chermahini F, Godderis L. Air pollution, environmental chemicals, and smoking may trigger vitamin D deficiency: Evidence and potential mechanisms. Environ Int. 2019;122:67-90.

29. Wimalawansa SJ, Razzaque MS, Al-Daghri NM. Calcium and vitamin D in human health: ¿Hype or real?. J Steroid Biochem Mol Biol. 2018;180:4-14.

30. Płudowski P, KarczmarewiczE, Bayer M, Carter G, Chlebna-Sokół $\mathrm{D}$, Czech-Kowalska J, et al. Practical guidelines for the supplementation of vitamin D and the treatment of deficits in Central Europe - recommended vitamin D intakes in the general population and groups at risk of vitamin D deficiency. Endokrynol Pol. 2013;64:319-27. 31. Spiro A, Buttriss JL. Vitamin D: An overview of vitamin D status and intake in Europe. Nutr Bull. 2014;39:322-50.

32. Vitamin D. Monograph. Altern Med Rev. 2008;13:153-64.

33. Pludowski P, Holick MF, Grant WB, Konstantynowicz J, Mascarenhas MR, Haq A, et al. Vitamin D supplementation guidelines. J Steroid Biochem Mol Biol. 2018;175:125-35.

34. Verma A, Vincent-Chong VK, DeJong H, Hershberger PA, Seshadri M. Impact of dietary vitamin D on initiation and progression of oral cancer. J Steroid Biochem Mol Biol. 2020;199:105603.

35. Haines ST, Park SK. Vitamin D supplementation: what's known, what to do, and what's needed. Pharmacotherapy. 2012;32:354-82.

36. Małodobra-Mazur M, Paduch A, Lebioda A, Konopacka M, Rogoliński J, Szymczyk C, et al. VDR gene single nucleotide polymorphisms and their association with risk of oral cavity carcinoma. Acta Biochim Pol. 2012;59:627-30.

37. Laczmanski L, Laczmanska I, Lwow F. Association of select vitamin D receptor gene polymorphisms with the risk of tobacco-related cancers - a meta-analysis. Sci Rep. 2019;5:16026. 
38. Li Q, Li Y, Jiang H, Xiao Z, Wu X, Zhang H, et al. Vitamin D suppressed gastric cancer cell growth through downregulating CD44 expression in vitro and in vivo. Nutr. 2021;15:111413.

\section{Funding}

None declared.

\section{Conflict of interest}

None declared.

\section{Authors contributions}

Andrea Maturana-Ramírez and Juan Aitken-Saavedra contributed equally.

All authors approved the final version of the manuscript. Conceptualization: (Juan Aitken-Saavedra, Andrea Maturana S); Methodology: (Juan Aitken-Saavedra, Andrea Maturana-Ramirez, Aranxta Ladron de Guevara-Benitez); Formal analysis and investigation: (Aranxta Ladron de Guevara-Benitez, Iris Espinoza-Santander); Writing - original draft preparation: (Juan Aitken-Saavedra, Andrea Maturana-Ramirez); Writing - review and editing: (Juan AitkenSaavedra, Andrea Maturana-Ramirez, Iris Espinoza-Santander), Supervision: (Andrea Maturana-Ramirez). 